\title{
Primary Actinomycosis of the Foot: A Case Report and Literature Review
}

\author{
Jacob Bettesworth, MD, ${ }^{a}$ Kirandeep Gill, MD, ${ }^{a}$ Jayesh Shah, MD ${ }^{b, *}$
}

\author{
${ }^{a}$ Kansas City School of Medicine and Biosciences, Kansas City, KS, USA; and \\ ${ }^{b}$ Southwest Center for Wound Care and Hyperbaric Medicine, Southwest General Hospital, San Antonio, TX, USA
}

\section{KEYWORDS:}

Actinomycosis;

Foot;

Hyperbaric oxygen

therapy;

Unusual wounds

\begin{abstract}
Actinomycosis is an indolent, slowly progressive, suppurative infection caused by gram-positive branching bacteria of the genus Actinomyces. The organism is a member of the oral and gastrointestinal microflora of humans. The disease actinomycosis most commonly occurs in 3 body regions: cervicofacial (55\% of patients), abdominopelvic (20\%), and pulmonothoracic (15\%). Involvement of other parts of the body is uncommon and usually secondary to a lesion in one or the other of the above sites. Extremity disease can occur by secondary involvement through direct extension or hematogenous spread. However, primary actinomycosis of an extremity is very rare. A case of primary actinomycosis of the foot in a Hispanic male and a literature review with an emphasis on primary actinomycosis of the extremity are presented in this article.
\end{abstract}

(C) 2009 Elsevier Inc. All rights reserved.

\section{Introduction}

Actinomycosis is an indolent, slowly progressive, suppurative infection caused by gram-positive branching bacteria of the genus Actinomyces. The organism is a member of the oral and gastrointestinal microflora of humans. The disease actinomycosis most commonly occurs in 3 body regions: cervicofacial (55\% of patients), abdominopelvic (20\%), and pulmonothoracic (15\%). ${ }^{1}$ Involvement of other parts of the body is uncommon and usually secondary to a lesion in one or the other of the above sites. Extremity disease can occur by secondary involvement through direct extension or hematogenous spread. However, primary actinomycosis of an extremity is very rare. A case of primary actinomycosis of the foot in a Hispanic male and a

Conflict of interest: The authors report no conflicts of interest.

* Corresponding author.

E-mail address: Jayeshneha@yahoo.com literature review with an emphasis on primary actinomycosis of the extremity are presented in this article.

\section{Case History}

A 42-year-old construction worker from Mexico was first seen in the wound center with a chief complaint of a left swollen foot slowly progressing over multiple years. He complains of multiple small wounds with purulent drainage on the plantar aspect of the left foot. The patient was in his usual state of health until approximately 3 years ago, when he noted some discoloration of the left plantar area. The swelling got progressively worse over approximately 2 years and had been unchanged during the past year. Before presenting to us, he had been seen in the past 6 months by several physicians, who treated him with oral antibiotics. The patient stated, from time to time he developed localized swelling in the left foot, usually on the lateral and metatarsal area, which he squeezed to obtain small amounts 
of purulent fluid that had mild foul odor at times. The patient also reports work-related repeated trauma to the left foot.

On examination, the patient was afebrile, and his systemic examination was negative. Local examination showed a massively swollen left foot from the metatarsal area to the toes. The skin was thick, hyperpigmented, and with a few dry fistulous tracts. The plantar area of foot had multiple small sinus lesions with some nodules, no foul odor, no areas of drainage, and no subcutaneous crepitus (Figure 1). Two fistulous tracts in the medial aspect of the heel were noted. There were no lymphatic streaks or palpable inguinal adenopathy. Peripheral pulses were present.

Laboratory findings included a white blood cell count of 8,200 , hematocrit of $38 \%$, a platelet count of 337,000, erythrocyte sedimentation rate of 50, and C-reactive protein of 1.91. Blood cultures were negative on 2 different occasions. Magnetic resonance imaging was read as showing chronic inflammation and hypertrophy of the skin and osteomyelitis with involvement of the midtarsals and distal tarsals, all of the metatarsals, and the first and second phalange of the foot. These changes were confirmed on a bone scan.

A biopsy of the left foot was stained with hematoxylin and eosin showing epidermal and soft tissue abscess formation, with many of the abscesses containing purple filamentous sulfur granules (Figure 2). The biopsy also showed sinus tract formation throughout the soft tissue and hemosiderin deposition within the dermis. No fungal organisms were identified on the Gomori's methenamine silver stain. Identification of the organism as Actinomyces israelii was made on the morphologic and biochemical characteristics. A culture of the biopsy with antibiotic sensitivity was found to be sensitive to penicillin.

Because of extensive osteomyelitis, a below-the-knee amputation was advised. However, the patient refused.

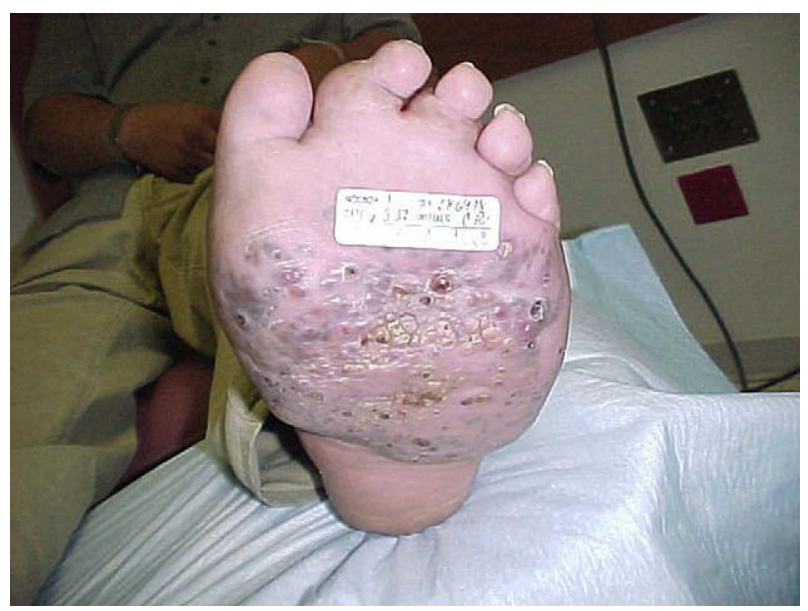

Figure 1 Patient With Actinomycosis Foot Infection With Multiple Sinuses.

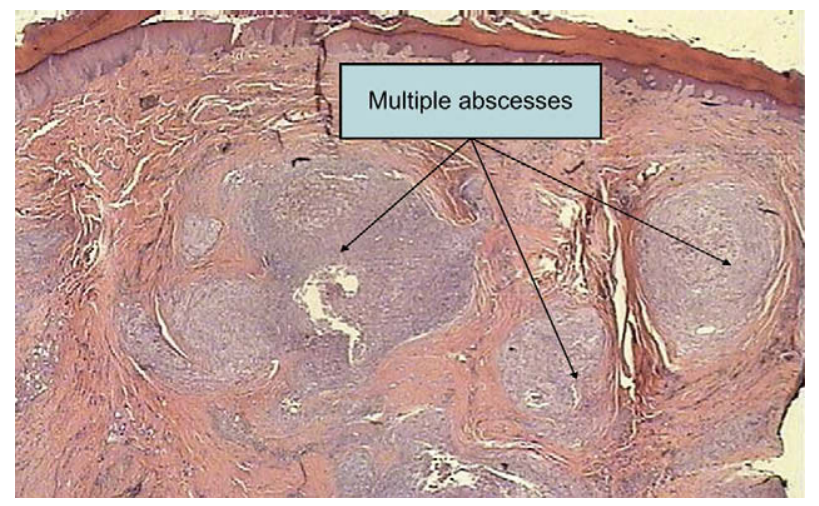

Figure 2 Hematoxylin and Eosin Stain of Patient's Tissue Biopsy From the Wound Showing Epidermal and Soft Tissue Abscess (Purulent Loculations) Formation With Many Purple Filamentous Sulfur Granules.

Thus, he was started on long-term amoxicillin antibiotic therapy and 30 treatments of hyperbaric oxygen. At completion of hyperbaric oxygen therapy, the patient had decreased exudate from the sinuses and was found to have improvement in swelling of the foot.

\section{Epidemiology}

The disease actinomycosis was formerly a common disease in humans (most common etiology, A. israelii) and cattle (A. bovis); however, as dental care has improved and antibiotics have become more widely used, the disease has become rare in the United States. ${ }^{1}$ For this reason, high-risk patients are those who do not seek or have access to health care. Early reports incorrectly classified actinomyces as an aerobic organism that could be cultured from the environment; however, it is now well accepted that actinomyces cannot be cultured from nature, and there are no documented cases of person-to-person transmission. ${ }^{2}$ One study found actinomyces in the oral mucous membranes in nearly $100 \%$ of infants by 2 years of age, ${ }^{3}$ whereas other studies have shown actinomyces to also be a member of the endogenous microflora of the gastrointestinal tract and female genital tract. ${ }^{4}$

Reports indicate that actinomycosis can occur in all ages, with a peak incidence occurring in the middle decades. It is well reported that males are infected more frequently than females, at a ratio of approximately 3 to 1. ${ }^{1,5,6,7,8}$ Explanations have postulated that males are more prone to oral trauma and have poorer dental hygiene. ${ }^{1}$

\section{Clinical Pathophysiology and Pathogenesis of Actinomycosis}

Actinomyces species are endogenous to the body and live normally within the human microflora mentioned earlier. 
The pathogenic form is parasitic and occurs when there is disruption of the mucosal barrier and the organism inoculates into soft tissues where there is impaired blood supply. ${ }^{1,9}$ Spread of the organism occurs through direct extension without regard to tissue planes, lymphatics, or hematogenous routes. Thus, systemic disease, extremity involvement, and regional adenopathy are not common manifestations. ${ }^{1}$ Pathogenesis may involve the ability of these organisms to suppress some of the immune functions of the host. Actinomyces have been shown to be chemotactic, to activate blastogenesis, and to stimulate the release of lysosomal enzymes from polymorphonuclear leukocytes and macrophages. ${ }^{9}$ However, when phagocytized by host defense cells, they cannot be killed and thus are defined as facultative intracellular parasites similar to Mycoplasma tuberculosis in their disease-causing role. ${ }^{1}$ This explains the strong tendency to cause chronic granulomatous inflammation of soft tissues and external sinuses, ${ }^{1}$ although the factor responsible for this unique pathogenesis has not been determined. ${ }^{9}$

Actinomyces, being saprophytes, do not produce exotoxins or significant amounts of other toxic substances. It is interesting that other bacteria have been cultured in as many as $99 \%$ of actinomycosis lesions, giving the other bacteria the name companion microbes. ${ }^{2,10}$ These other bacteria frequently consist of anaerobic streptococci, fusiform bacilli, hemophilus species, and various gram-negative bacilli. Actinomyces and the companion microbes are apparently synergistic in their relationship and are found in both open and closed lesions, suggesting the possibility of either primary or secondary infection. Since studies have proven that pure actinomyces inoculation of laboratory animals does not produce disease, it is postulated that these associated bacteria create an anaerobic environment allowing actinomyces to thrive and grow. ${ }^{1}$

The lesions usually appear as single or multiple indurated swellings. As the swellings mature, purulent loculations develop, and central suppuration causes the swellings to become soft (Figure 2). Microscopically, lesions consist of a dense outer zone of granulation tissue made up of collagen and fibroblasts. The granulation tissue forms multiple loculations with a purulent center containing sulfur granules surrounded by neutrophils, microcolonies of actinomyces, cellular debris, and other associated microorganisms (Figures 3 and 4). The sulfur granules are virtually diagnostic. They range in size from microscopic to macroscopic, and each loculation contains 1 to 6 sulfur granules. $^{2}$

On physical examination, the fibrous walls of the lesion may be characterized as "wooden," and if central suppuration does not occur, they are frequently confused with neoplasms. ${ }^{11}$ The skin often assumes a bluish or purplish to red hue. With time, sinus tracts develop and extend to adjacent tissue, eg, skin, bone, or organs, depending on the location. These sinus tracts can spontaneously open or close elsewhere. $^{2}$

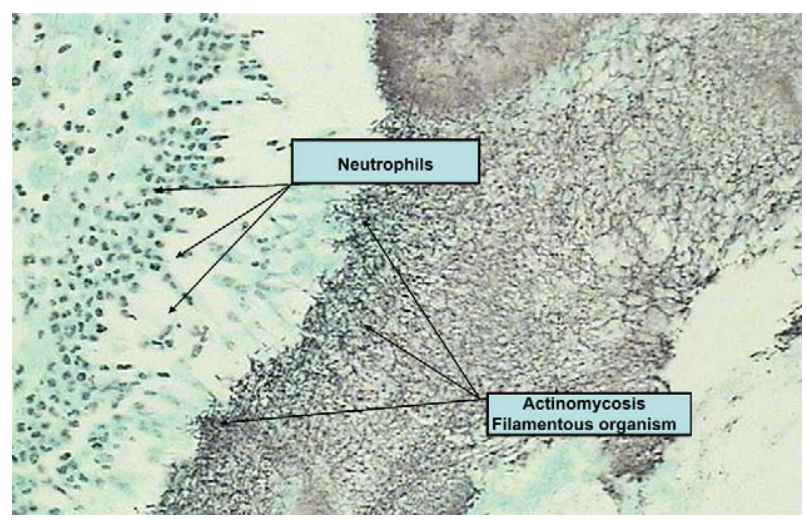

Figure 3 Hematoxylin and Eosin Stain of Tissue Biopsy Obtained From Patient's Wound and Illustrating Inflammatory Cells (Neutrophils) and Filamentous Actinomycosis Organisms.

\section{Reported Clinical Manifestation}

As stated above, actinomycosis is most commonly reported in 3 body regions: cervicofacial, abdominopelvic, and pulmonothoracic; however, other rare presentations have also been reported. Hematogenous dissemination can occur from the common primary sites and is reported to occur in $3 \%$ of cases. ${ }^{12}$ The most frequent source of hematogenous spread is reported to be from initial pulmonary infection, following aspiration of contents. ${ }^{13}$ Actinomycosis infections have also occurred in surgical- and medicalrelated foreign bodies. Zaman et al have identified cases of actinomycosis in infected hip prosthesis patients with a recent dental extraction in 2 of the cases and a history of IV drug abuse in 1 case. $^{14,15}$ Also, the epidemiologic association between use of an intrauterine device and pelvic actinomyces infections has been clearly demonstrated in several studies. ${ }^{16}$

Pang et $\mathrm{al}^{12}$ note that since the first report of anaerobic osteomyelitis in 1844 by Von Langenbeck, 850 cases have been reported. One percent of all reported cases had osteomyelitis with actinomyces. Only 28 cases of

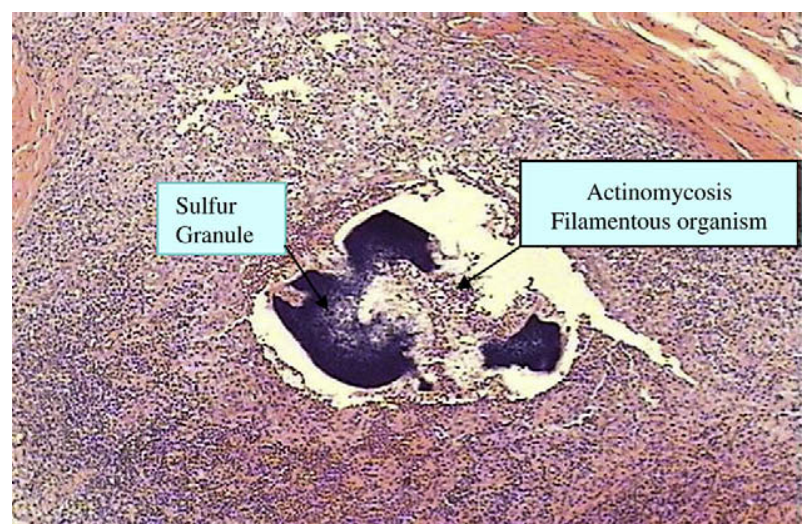

Figure 4 Tissue Biopsy of the Wound Containing Both Sulfur Granules and Actinomycosis Filamentous Organisms. 
osteomyelitis of the extremity have been reported in the literature, with 11 occurring in the hand and foot. ${ }^{17}$ Pang et al also note only 1 reported case ${ }^{18}$ in the English literature of osteomyelitis of the foot confirmed by culture as Actinomyces israelii. ${ }^{12}$

Primary actinomyces of an extremity is very uncommon because of the endogenous habitat of the organism. Reiner et al report that only 36 cases of primary actinomycosis of the extremity have occurred in the past 107 years. Of the 36 reported cases, 21 had a history of perforating trauma, mostly due to oral cutaneous contact such as a biting injury or punch to the mouth. Of the other 15 cases, with no clear trauma history, hematogenous spread is suggested in 7 , and the other 8 cases may have had a traumatic injury due to the patient's occupation but could not be documented. Only 4 reported cases in the last 99 years had foot involvement. ${ }^{8}$ One of the 4 had a remote history of foot trauma, ${ }^{18}$ and in the second case, the patient's occupation of farmer in Sudan may suggest an unknown posttraumatic cause of infection. ${ }^{19}$ The third case had a possible hematogenous source, ${ }^{20}$ while in the final case, there was no suggested source of infection by history. ${ }^{21}$

Two additional cases of primary foot actinomycosis have been reported in the nearly 20 years since the Reiner et al report in 1986. The case of a 50-year-old male with a questionable traumatic etiology and a history of severe gingivitis, who developed osteomyelitis of the foot, was reported in 1987. Cultures of the foot lesion demonstrated Actinomyces meyeri. ${ }^{12}$ The most recent report, in 1996, was of a 34-year-old female with a 7-year history of painless swelling of her left foot, fourth interspace. The patient preferred to walk around the house and yard with bare feet; however, no trauma or laceration was reported. The mass was removed surgically, and the pathologist's microscopic evaluation describes Actinomyces species. ${ }^{22}$

\section{Diagnosis}

Actinomycosis is a difficult disease to diagnose. ${ }^{23}$ Three important clinical presentations should prompt this diagnosis: (1) the chronicity of a masslike lesion that extends through tissue borders and mimics a tumor, (2) the development of sinus tracts that spontaneously resolve and recur, and (3) a relapsing infection following a short course of antibiotics. $^{2}$

A correct diagnosis requires a combination of microbiological, molecular, and pathologic studies. A clinical diagnosis starts with obtaining a sample of suppurative exudate, tissue, or sulfur granules (Figure 4). The literature has described many ways to obtain a sample, depending on the location, such as computed tomography-guided core biopsies of the pelvis ${ }^{24}$ and ultrasound-guided fine needle aspiration of the thorax. ${ }^{25}$ It is extremely helpful if the sample demonstrates grains in the purulent drainage or histologic section of a surgical specimen. ${ }^{2}$ The grains represent infection that occurs in vivo and are merely a conglomeration of microorganisms. A hematoxylin and eosin stain is used to demonstrate the grain, but a Gram or silver stain is used to show that the grain is composed of branching bacteria (Figure 5). It is imperative that antibiotic therapy be withheld until the sample specimen is obtained; otherwise, actinomyces may not be isolated. If antibiotics have been given already, Gram stain is usually more sensitive than culture. A diagnosis of actinomycosis is considered whenever a direct Gram stain of the suppurative exudate shows gram-positive, non-acid-fast rods in diphtheroidal arrangements with or without branching. $^{2}$

Actinomyces can be cultured on a rich medium, such as brain heart infusion blood agar, and incubated anaerobically with added carbon dioxide. The source of actinomyces used for culture are taken from crushed granules or well-mixed pus in the absence of granules. ${ }^{9}$ The growth usually appears within 5 to 7 days, but primary isolation may take up to 2 to 4 weeks. Traditional identification depends on the combination of isolates: morphologically grown patterns resembling actinomyces species; the tests for urease, catalase, gelatin hydrolysis; and fermentation of cellobiose, trehalose, and arabinose. ${ }^{2}$

Many other tests can assist in the diagnosis, such as determination of the metabolic end products by gas-liquid chromatography. ${ }^{26}$ Immunofluorescence tests are useful for serologic identification of isolates and the direct demonstration of the organisms in clinical samples. ${ }^{9}$ Ambiguous results with non-spore-forming, gram-positive rods can be resolved by sequencing or using restriction analysis of amplified 16S ribosomal DNA. ${ }^{27}$ Holmberg $^{28}$ highlights various serologic assays that can be used in the diagnosis of actinomyces; however, improvements in sensitivity and specificity are necessary before these assays can be useful in clinical practice. ${ }^{2}$

The diagnosis of actinomycosis, especially in the extremity, must be separated from actinomycetoma and botryomycosis. Botryomycosis is a chronic bacterial infection of soft tissues that produces loose clumps of bacteria

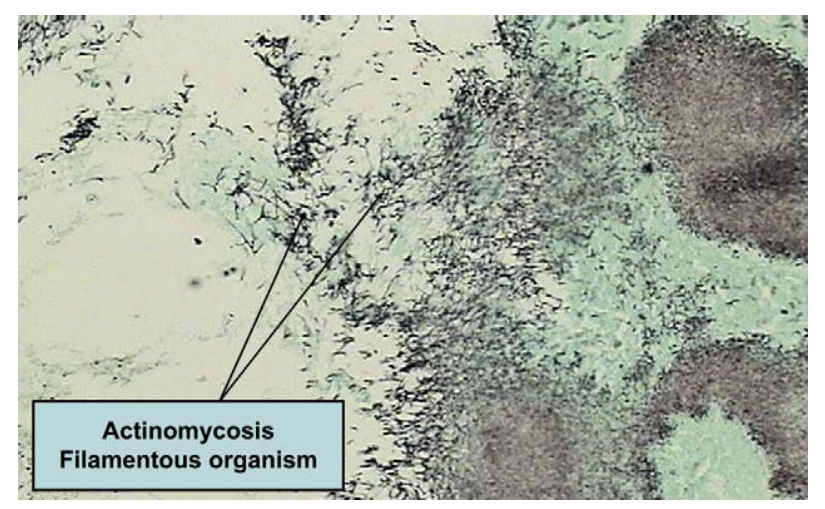

Figure 5 Tissue Biopsy Illustrating Actinomycosis Filamentous Organisms. 
resembling granules. It is easily separated from actinomycosis by the presence of cocci or nonbranching bacilli. Actinomycetoma is a form of madura foot or mycetoma and caused by nocardia species. ${ }^{8}$ Actinomycosis of an extremity and actinomycetoma can produce virtually the same disease characteristics, ie, localized chronic and progressive infection with purulent sinus tract drainage containing granules and involvement of contiguous bone. The 2 diseases are considered distinct and separate clinical entities because of their differences in pathogenesis and habitat. ${ }^{29}$ Histologically, on Gram stain they may be indistinguishable; however, Fite modified acid-fast will stain nocardia species but not actinomyces. ${ }^{2}$ When the etiology of a granule is in question, specific immunofluorescent stains can be used to isolate the various actinomyces species. $^{28}$ The correct diagnosis of actinomycosis versus actinomycetoma is important because they are sensitive to different antibiotic treatments. ${ }^{8}$

\section{Treatment}

The drug of choice in the treatment of actinomycosis continues to be penicillin. The principle of therapy began with Peabody and Seabury back in 1960. They have shown that because of the tendency of actinomyces to recur following apparently successful treatment, penicillin is necessary in high doses and for prolonged periods of time. This allows the antibiotic to penetrate the avascular fibrotic walls of the lesion and reach the core of the sulfur granules. ${ }^{30}$ Therapy must be individualized, although complicated infections usually require a 2-to-6-week course of 18 million to 24 million units parenteral penicillin $\mathrm{G}$ followed by a prolonged course of oral penicillin $\mathrm{V}$ or amoxicillin for 6 to 12 months. It is a reasonable approach to also include antibiotic coverage for companion microbes in the initial treatment because many are pathogens on their own. $^{2}$

For penicillin-allergic patients, alternatives such as tetracycline, doxycyline, erythromycin, and clindamycin may be given. ${ }^{31}$ Newer agents such as imipenem, ${ }^{32}$ ceftriaxone, ${ }^{33}$ and ciprofloxacin ${ }^{34}$ have been used with anecdotal success. Antimicrobial agents that are not effective against actinomyces are oxacillin, dicloxacillin, cephalexin, metronidazole, and aminoglycosides. ${ }^{35}$

A combination of antimicrobial and surgical treatments is used in the treatment of actinomyces infection. Some data indicate that medical therapy alone may be all that is needed, even with extensive disease. ${ }^{36,37}$ Radiology modalities such as computed tomography or magnetic resonance imaging can be used to monitor therapy success. ${ }^{38}$ In the case of a well-defined abscess, percutaneous drainage is a reasonable option along with medical therapy. ${ }^{39} \mathrm{~A}$ few case reports suggest hyperbaric oxygen therapy may be used as an adjunct with antimicrobial and surgical treatment in refractory actinomycosis infections. ${ }^{40,41}$
Treatment recommendations are limited because of unavailability of an evidence-based protocol for chronic actinomycosis.

\section{References}

1. Bennhoff D: Actinomycosis: diagnostic and therapeutic considerations and a review of 32 cases. Laryngoscope. 1984;94:1198-1217.

2. Russo TA: Agents of actinomycosis. In: Mandell GL, Bennet JE, Dolin R, editors. Principles and Practice of Infectious Diseases. 6th ed. New York, NY: Churchill Livingstone, 2005. p. 2925-2932.

3. Sarkonen N, Kononen E, Summanen P, et al: Oral colonization with actinomyces species in infants by two years of age. J Dent Res. 2000;79:864-867.

4. Persson E: Genital actinomycosis and Actinomyces israelii in the female genital tract. Adv Contracept. 1987;3:115-123.

5. Harvey J, Cantrell J, Fisher A: Actinomycosis: Its recognition and treatment. Ann Intern Med. 1957;46:868-885.

6. Kinnear W, MacFarlane J: A survey of thoracic actinomycosis. Respir Med. 1990;84:57-59.

7. Spilsbury BW, Johnstone FRC: The clinical course of actinomycotic infections: a report of 14 cases. Can J Surg. 1962;5:33-48.

8. Reiner SL, Harrelson JM, Miller SE, Hill GB, Gallis HA: Primary actinomycosis of an extremity: a case report and review. Rev Infect Dis. 1987;9(3):581-589.

9. Bowdon GHW. Actinomyces, Propionibacterium propionicus, and streptomyces. In: Baron S, ed. Medical Microbiology. 4th ed. Available at: http://www.ncbi.nlm.nih.gov/books/bv.fcgi?rid=mmed. chapter.1863. Accessed July 6, 2009.

10. Holm P: Studies on etiology of human actinomycosis. I. The "other" microbes of actinomycosis and their importance. Acta Pathol Microbiol Scand. 1950;27:736.

11. Colmegna I, Rodriguez-Barradas M, Rauch R, Clarridge J, Young EJ: Disseminated Actinomyces meyeri infection resembling lung cancer with brain metastases. Am J Med Sci. 2003;326(3):152-155.

12. Pang DK, Abdalla M: Osteomyelitis of the foot due to Actinomyces meyeri: a case report. Foot Ankle. 1987;8(3):169-171.

13. Van Mook WN, Simonis FS, Schneeberger PM, van Opstal JL: A rare case of disseminated actinomycosis caused by Actinomyces meyeri. Neth J Med. 1997;51(1):39-45.

14. Zaman R, Abbas M, Burd E: Late prosthetic hip joint infection with Actinomyces israelii in an intravenous drug user: case report and literature review. J Clin Microbiol. 2002;40(11):4391-4392.

15. Wüst J, Steiger U, Vuong H, Zbinden R: Infection of a hip prosthesis by Actinomyces naeslundii. J Clin Microbiol. 2000;38:929-930.

16. Lee YC, Min D, Holcomb K, Buhl A, DiMaio T, Abulafia O: Computed tomography guided core needle biopsy diagnosis of pelvic actinomycosis. Gynecol Oncol. 2000;79(2):318-323.

17. Lewis RP, Sutter VL, Finegold SM: Bone infections involving anaerobic bacteria. Medicine (Baltimore). 1978;57:279-305.

18. Mohanty PR, Parija SC, Patra DK, Yadav SS, Chandra S, Valiath AJ: Actinomycosis involving bone: report of a case. Indian J Pathol Microbiol. 1983;26(4):321-323.

19. Mahgoub ES, Yacoub AA: Primary actinomycosis of the foot and leg: report of a case. J Trop Med Hyg. 1968;71(10):256-258.

20. Cope VZ: Actinomycosis. London, England: Oxford University Press; 1938.

21. Buerger L. Actinomycosis of the skin of the foot. Am J Med Sci. 1907; 134:702-709.

22. Vogen KW. Actinomycosis of the foot. J Am Podiatr Med Assoc. 1996;86(5):238-240.

23. Cope Z. Visceral actinomycosis. Br Med J. 1949;2(4640):1311-1316.

24. Lee YC, Min D, Holcomb K, Buhl A, DiMaio T, Abulafia O: Computed tomography guided core needle biopsy diagnosis of pelvic actinomycosis. Gynecol Oncol. 2000;79(2):318-323. 
25. Hsu WH, Chiang CD, Chen CY, Hsu JY, Chang MC: Ultrasoundguided fine needle aspiration biopsy in the diagnosis of chronic pulmonary infection. Respiration. 1997;64(5):319-325.

26. Sarkonen N, Kononen E, Summanen P, et al: Phenotypic identification of actinomyces and related species isolated from human sources. $\mathrm{J}$ Clin Microbiol. 2001;39:3955-3961.

27. Sato T, Matsuyama J, Takahashi N, et al: Differentiation of oral actinomyces species by 16 S ribosomal DNA polymerase chain reaction-restriction fragment length polymorphism. Arch Oral Biol. 1998;43:247-252.

28. Holmberg K. Diagnostic methods for human actinomycosis. Microbiol Sci.. 1987;4:72-78.

29. Tight RR, Bartlett MS: Actinomycetoma in the United States. Rev Infect Dis. 1981;3:1139-1150.

30. Peabody J, Seabury J: Actinomycosis and nocardiosis: a review of basic differences in therapy. Am J Med. 1960;60:99-115.

31. Martin MV. Antibiotic treatment of cervicofacial actinomycosis for patients allergic to penicillin: a clinical and in vitro study. Br J Oral Maxillofacial Surg. 1985;23:428-435.

32. Yew W, Wong P, Wong C, Chau C: Use of imipenem in the treatment of thoracic actinomycosis. Clin Infect Dis. 1994;19:983-984.
33. Skoutelis A, Petrochilos J, Bassaris H: Successful treatment of thoracic actinomycosis with ceftriaxone. Clin Infect Dis. 1994;19:161-162.

34. Macfarlane D, Tucker G, Kemp R: Treatment of recalcitrant actinomycosis with ciprofloxacin. J Infect. 1993;27:177-180.

35. Shore KP, Pottumarthy S, Morris AJ: Susceptibility of anaerobic bacteria in Auckland: 1991-1996. N Z Med J. 1999;112:424-426.

36. Marty H, Wust J: Disseminated actinomycosis caused by Actinomyces meyeri Infection. 1989;17:154-155.

37. Schleck W, Gelfand M, Alper B, et al: Medical management of visceral actinomycosis. South Med J. 1983;76:921-922.

38. Hawnaur JM, Reynolds K, McGettigan C: Magnetic resonance imaging of actinomycosis presenting as pelvic malignancy. Br J Radiol. 1999;72:1006-1011.

39. Goldwag S, Abbitt P, Watts B: Case report: percutaneous drainage of periappendiceal actinomycosis. Clin Radiol. 1991;44:422-442.

40. Manheim SD, Voleti C, Ludwig A, Jacobson JH II: Hyperbaric oxygen in the treatment of actinomycosis. JAMA. 1969;210(3):552-523.

41. Shauly Y, Nachum Z, Gdal-On M, Melamed Y, Miller B: Adjunctive hyperbaric oxygen therapy for actinomycotic lacrimal canaliculitis. Graefes Arch Clin Exp Ophthalmol. 1993;231(7):429-431. 